records. Mortality data were derived by linking with data from the Korean National Statistics Office (KNSO). Multivariable logistic regression and cox regression test were performed to assess the risk factor of NPSLE and predictors of mortality. Results Of 1121 SLE patients, 429 (38.2\%) patients had NPSLE events according to ACR definitions and 216 (19.3\%) by Ainiala criteria. In multivariable logistic regression analysis, year of education [Odds ratio (OR) 0.92, 95\% confidence interval (CI) 0.87 to $0.96, \mathrm{p}<0.01$ ] and elevated anti-dsDNA antibodies (OR 0.52 , CI: 0.37 to $0.76, p<0.01$ ) decreased the risk of NPSLE. In multivariable cox regression analysis, SLEDAI without NP manifestations at enrollment increased the risk of mortality (OR 1.18, CI: 1.08 to $1.25, \mathrm{p}<0.01$ ) in NPSLE patients.

Conclusion The $38.2 \%$ and $19.3 \%$ of SLE patients had NPSLE according to ACR and Ainiala definition of NPSLE. Year of education and elevated anti-dsDNA antibodies decreased the risk of occurrence of NPSLE. SLEDAI without NP manifestations at enrollment increased the risk of mortality in NPSLE patients.

\section{PS3:54 CHARACTERISTIC FEATURES OF HAEMATOLOGICAL INVOLVEMENT AND ITS EFFECT ON DAMAGE ACCRUAL IN PATIENTS WITH SYSTEMIC LUPUS ERYTHEMATOUSUS: PRELIMINARY RESULTS FROM A MULTICENTER EUROPEAN COHORT}

${ }^{1} \mathrm{~S}$ Yavuz, ${ }^{2} \mathrm{D}$ Cansu, ${ }^{3} \mathrm{~F}$ Crisafulli, ${ }^{4} \mathrm{AM}$ Antunes, ${ }^{5} \mathrm{D}$ Nikolopoulos, ${ }^{6} \mathrm{~K}$ Tascilar, ${ }^{2} \mathrm{C}$ Korkmaz,
${ }^{3} \mathrm{~L}$ Andreoli, ${ }^{4} \mathrm{~F}$ Moraes-Fontes, ${ }^{3} \mathrm{~A}$ Tincani, ${ }^{5} \mathrm{G}$ Bertsias. ${ }^{1}$ Department of Rheumatology
Istanbul Bilim University, Istanbul, Turkey; ${ }^{2}$ Department of Rheumatology Osmangazi
University, Eskisehir, Turkey; ${ }^{3}$ Rheumatology and Clinical Immunology Unit,Spedali Civili and
University of Brescia, Italy; ${ }^{4}$ Unidade de Doencas Autoimmunes/Servico Medicina7.2
Hospital Curry Cabral, Centro Hospitalara de Lisboa Central, Lisboa, Portugal;
${ }^{5}$ Rheumatology and Clinical Immunology Unit 4th Department of Internal Medicine Attikon
University Hospital, Athens, Greece; ${ }^{6}$ Saglik Bilimleri University Okmeydani Egitim ve
Arastirma Hastanesi IC Hastaliklari, Istanbul, Turkey

\subsection{6/lupus-2018-abstract.101}

Background and aim We studied haematological manifestations (HM) and their impact on the progression of damage in systemic lupus erythematosus (SLE) using a multicenter European cohort of patients.

Methods We examined the observational data of a SLE patients with serial clinical and laboratory measurements of every 6 months for 2 years gathered from 4 different countries. Each collaborative centre was asked for a contribution of fifty or more consecutive SLE patients. We compared clinical features, antibody profiles, SLEDAI-2K and SDI in patients with and without HM using Chi-Square and Student's t-tests for categorical and continuous variables, respectively. Multivariate Cox Proportional hazards regression was used the investigate the quartiles of leukocytes, lymphocytes and platelets at every time point (at 0,6,12,18,24 months) in relation to the damage characterised by the SDI scores. Probability of change in damage index (from SDI $=0$ to SDI equal or greater than 1) was calculated using mixed models logistic regression. Adjustments ma Results are presented as odds ratios (ORs) with their 95\% CIs; results were defined significant as a $\mathrm{p}$ 0.05 .

Results So far, 751 measurements of 159 patients were examined. Mean age was 44.9 (13.5) vs 44.0 (12.9) for patients with and without $\mathrm{HM}$, respectively $(\mathrm{p}=\mathrm{NS})$. Mean disease duration at the time of cohort created was 11.1 (6.2) vs 10.8
(4.9) in patients with or without HM. Demographic features, clinical characteristics of patients with HM at SLE diagnosis or during the follow up are demonstrated in table 1 . Sex, ethnicity and baseline autoantibodies showed no influence on damage. SLEDAI-2K was associated with an increased OR of 2.1 [95\% CI: 1.29 to 3.42] for damage.

Conclusion Preliminary results imply that disease activity predicts future damage accrual in patients with haematological manifestations.

\begin{tabular}{|c|c|}
\hline Characteristics & $\begin{array}{l}\text { Median (range) unless stated } \\
\text { otherwise }\end{array}$ \\
\hline Gender: female, $n(\%)$ & $101(88)$ \\
\hline \multicolumn{2}{|l|}{ Ethnicity, $O(56)$} \\
\hline Caucasian & $109(94)$ \\
\hline African & 6(6) \\
\hline \multicolumn{2}{|l|}{ SDI first recorded, $0(26)$} \\
\hline 0 & $90(78)$ \\
\hline 1 & $18(16)$ \\
\hline 22 & $7(6)$ \\
\hline \multicolumn{2}{|l|}{ HM first detected } \\
\hline Disease onset & $78(68)$ \\
\hline Disease course & $37(32)$ \\
\hline Leukopenia $\leq 3000$ & $27(23\}$ \\
\hline Lymphopenia s 1000 & $71(62)$ \\
\hline Thrombocytopenia & $39(34)$ \\
\hline AHA & $20(17)$ \\
\hline \multicolumn{2}{|c|}{ Associated clinical features } \\
\hline Musculoske letal & $75(65)$ \\
\hline Cutaneous & $57(50)$ \\
\hline Renal & $33(29)$ \\
\hline Neurological & $12(10)$ \\
\hline \multicolumn{2}{|l|}{ Associated Abs } \\
\hline Anti-dsDNA & $60(53)$ \\
\hline Anti-Ro & $31(27)$ \\
\hline Anti-Sm & $18\{16)$ \\
\hline
\end{tabular}

\section{PS3:55 PREDICTIVE POTENTIAL OF THE DISEASE ACTIVITY INDEX AND C-REACTIVE PROTEIN FOR INFECTION IN SYSTEMIC LUPUS ERYTHEMATOSUS PATIENTS}

${ }^{1}$ SMG Sherif, 'S Fakhreldin, ${ }^{2} \mathrm{AS}$ Saad. ${ }^{1}$ Faculty of Medicine, Cairo University, Cairo, Egypt; ${ }^{2}$ Shobra General Hospital, Cairo, Egypt

10.1136/lupus-2018-abstract.102

Aim of the work The aim of the present work was to determine the prevalence of infections in a cohort of Egyptian Systemic lupus erythematosus (SLE) patients and to describe their 
sites and relation to clinical characteristics, laboratory features and disease activity.

Patients and methods Medical records of 250 Egyptian SLE patients attending the Rheumatology department, Cairo University hospitals were reviewed retrospectively for the clinical and laboratory features, SLE disease activity index (SLEDAI) and treatment received.

Results Infection was found in 119 (47.6\%) patients, with bacterial infection being the commonest in 99 (83\%) followed by fungal infection in $30(25 \%)$ and viral infection in 22 (18.5\%). The commonest site of infection was the skin (37\%) followed by the urinary tract (31\%) and chest (19\%). In SLE patients with infection there was a significant increase in the frequency of malar rash $(p=0.001)$, photosensitivity $(p=0.01)$, oral ulcers $(p<0.001)$, alopecia $(p=0.017)$ and Raynauds $(p=0.017)$ compared to those without infection. Pulmonary and neuropsychiatric manifestations were also significantly increased in those with infection $(p=0.001$ and $p<0.001)$. A significantly higher number of patients with infection were receiving pulse steroids $(\mathrm{p}=0.016)$, cyclophosphamide $(p=0.011)$ and a higher oral prednisolone dose $(p=0.03)$. The SLEDAI was significantly higher $(26.02 \pm 8.23)$ in those with infection compared to those without $(15.57 \pm 6.43)(\mathrm{p}<0.001)$. $\mathrm{C}$-reactive protein (CRP) was significantly higher in those with infection $(p<0.001)$. On performing a logistic regression analysis, only SLEDAI $(\mathrm{p}<0.001)$ and CRP $(\mathrm{p}<0.001)$ were significant predictors of infection.

Conclusion Disease activity and CRP are important predictors for infection in SLE patients.

\section{PS3:56 THE ASSOCIATION BETWEEN SELF-REPORTED PHYSICAL ACTIVITY ON DISEASE STATUS PATIENTS WITH SYSTEMIC LUPUS ERYTHEMATOSUS: DATA FROM KOREAN LUPUS NETWORK (KORNET) REGISTRY}

S Kim, J Choe, S Park, H Lee. Catholic University of Daegu School of Medicine, Daegu, South Korea

\subsection{6/lupus-2018-abstract.103}

Objective The aim of this study was to identify the influence of physical activity on disease activity and damage in systemic lupus erythematosus (SLE).

Methods A total of 464 patients with Sjögren's syndrome were consecutively enrolled from KORean lupus Network (KORNET) registry. This registry assessed clinical features, disease activity (Systemic Lupus Erythematosus Disease Activity Index 2000 [SLEDAI-2K]), and disease damage (Systemic Lupus International Collaborating Clinics/American College of Rheumatology [SLICC/ACR] damage index) at the enrollment of study. Self-reported physical activity was measured by international physical activity questionnaire (IPAQ). Statistical analyses were used by Spearman's correlation and Mann-Whitney U test.

Results The median total physical activity (MET-minute/day) was 1173.0 (IQR 396.0-2772.0). There is significant difference of vigour activity between patients with lupus nephritis $(n=110)$ and without lupus nephritis $(n=354)(p=0.048)$, but not total, moderate, and walking activities. Among total patients, total IPAQ score was marginally associated with SLEDAI and SLICC/ACR scores $(r=-0.142, \quad \mathrm{p}=0.008$ and $r=-0.104, p=0.026)$. Higher SLICC/ACR scores was associated with lower walking activity and total activity of IPAQ in patients with lupus nephritis, $(\mathrm{r}=-0.256, \mathrm{p}=0.007$ and $\mathrm{r}=-0.193, \mathrm{p}=0.044$, respectively).

Conclusion This study showed that self-reported physical activity might be in part associated with disease activity and damage in patients with SLE.

\section{PS3:57 IMPACT OF LUPUSNEPHRITIS ON MORTALITY IN SYSTEMIC LUPUS ERYTHEMATOSUS. A POPULATION BASED COHORT FROM NORWAY}

${ }^{1}$ SER Moe, ${ }^{1} \varnothing$ Molberg, ${ }^{2} \mathrm{EH}$ Strøm, ${ }^{1} \mathrm{~K}$ Lerang. 'Department of Rheumatology, Os/o University Hospital, Rikshospitalet, Oslo, Norway: ${ }^{2}$ Department of Pathology, Oslo University Hospital, Rikshospitalet, Oslo, Norway

\subsection{6/lupus-2018-abstract.104}

Objective Recent data has shown that patients with lupus nephritis (LN) have increased mortality. However, no studies have been population based, and few compare mortality data in Systemic Lupus Erythematosus (SLE) patients with and without LN. The aim of our study was to investigate mortality in patients who does or does not develop LN in a population based cohort.

Methods Multiple sources were used to identify all SLE patients in Oslo during 1999-2009 who met 4 or more of the American College of Rheumatology (ACR) criteria. Follow up time was until 1 st January 2014. Presence of LN was defined by the ACR criteria. Standardised mortality ratio (SMR) was compared to observed deaths in a matched control population.

Results Of the 325 SLE patients included in this study, 98 (30\%) developed LN. 75 patients $(77 \%)$ had biopsy proven LN. A total of 56 deaths occurred during the study period, corresponding to an overall SMR for all SLE patients of 2,1 (95\% confidence intervall (CI) 1,2 to 3,4). The SMR estimate for LN patients were 3,8 (95\% CI: 2,1 to 6,2$)$ and $1,7(95 \%$ CI: 0,9 to 2,7$)$ for non $\mathrm{LN}$ patients.

Conclusion LN is associated with increased mortality, however SLE patients who do not develop LN have a good overall prognosis with no significant higher mortality than the general population.

\section{PS3:58 LIPID PROFILE CHARACTERISATION IN PATIENTS WITH JUVENILE SLE WITH AND WITHOUT LUPUS NEPHRITIS - EXPERIENCE OF A PORTUGUESE CENTRE}

\begin{abstract}
${ }^{1,2} \mathrm{~A}$ Águeda, ${ }^{3} \mathrm{M}$ Guerra, ${ }^{4} \mid$ Jorge, ${ }^{2,5} \mathrm{R}$ Ferreira, ${ }^{2,6} \mathrm{M}$ Rodrigues, ${ }^{2,6}$ । Brito. ${ }^{1}$ Rheumatology, Centro Hospitalar do Baixo Vouga E.P.E., Aveiro, Portugal; ${ }^{2}$ Faculty of Medicine of Porto University, Porto, Portugal; ${ }^{3}$ Rheumatology, Centro Hospitalar Vila Nova de Gaia/Espinho, Porto, Portugal; ${ }^{4}$ Physical Medicine and Rehabilitation, Centro Hospitalar do Porto, Portugal; ${ }^{5}$ Rheumatology, Centro Hospitalar de São João, Porto, Portugal; ${ }^{6}$ Pediatric Rheumatology Unit, Porto, Portugal

\subsection{6/lupus-2018-abstract. 105}

Introduction SLE is a multisystem chronic inflammatory disease and has been associated with premature atherosclerosis and so, controlling classic cardiovascular (CV) risk factors is crucial. Dyslipidaemia is an important $\mathrm{CV}$ risk factor and has been found to be altered in SLE patients.

Purpose Compare lipid profiles of two groups of Juvenile SLE patients, without Lupus Nephritis (group 1) and with Lupus Nephritis (group 2). Verify factors that might correlate with abnormalities in lipid profile. 